\title{
Photoacoustic technique: a new method for measuring the rheologic properties of human bronchial mucus
}

\section{Tecnica fotoacustica: un nuovo metodo per studiare le proprietà reologiche del muco bronchiale umano}

\author{
Luis V.F. De Oliveira ${ }^{1}$, Newton S. De Faria Jr. ${ }^{1}$, Fernanda M. Garcia Gonzaga Napoleone², \\ Marcelo A. Ingraci Barboza ${ }^{3}$, Fernando S. Studart Leitão Filho ${ }^{4}$, Luiz C. De Mattos ${ }^{3}$, \\ Claudio F. Donner ${ }^{5}$, Paulo R. Barja² \\ 'Sleep Laboratory, Rehabilitation Sciences Master and Doctoral Degree Program, Nove de Julho University, UNI- \\ NOVE, Sao Paulo, SP, Brazil \\ ${ }^{2}$ Research and Development Institute, Universidade do Vale do Paraíba, UNIVAP, Sao Jose dos Campos, SP, Brazil \\ ${ }_{3}^{3}$ Molecular Biology Department, Faculdade de Medicina de Sao Jose do Rio Preto, FAMERP, Sao Jose do Rio Preto, \\ SP, Brazil \\ ${ }^{4}$ Medicine School, Universidade de Fortaleza, UNIFOR, Fortaleza, CE, Brazil. \\ ${ }^{5}$ Mondo Medico, Multidisciplinary and Rehabilitation Outpatient Clinic, Borgomanero (NO), Italy
}

\section{INTRODUCTION}

Patients affected by obstructive pulmonary diseases exhibit typical characteristics of airway mucus hypersecretion, including sputum production, increased luminal mucus, goblet cell hyperplasia and submucosal gland hypertrophy. The mucociliary system is a sophisticated defense mechanism to maintain the homeostasis of the respiratory tract by means of the bronchial mucus transport, the process in which airway mucus together with substances trapped within are moved out of the lungs. In the end, the mucus together with the substances trapped within are swallowed or coughed up. It is an important defense mechanism of the human body [1].

Mucociliary clearance is influenced by viscosity and elasticity, that are the fundamental rheologic properties of the respiratory mucus and important determinants of its transportability in the mucociliary system [2]. It has been observed that the rheologic properties of mucus are often abnormal in patients with airways disease [3]. A retardation in mucociliary transport may lead to mucus retention, a condition which predisposes to bacterial colonization and may eventually lead to pneumonia. Drugs may alter the process of mucociliary transport, such that it is necessary to know the effect of the drugs on the rheologic properties of human bronchial mucus.

Understanding the interaction of a specific drug with human bronchial mucus may lead to a better therapeutic use of such medication. The photoacoustic technique (PAT) is a new method that permits to monitor indirectly the rheologic properties of any mucus sample $[3,4]$. PAT enables to study the interaction between therapeutic aerosols and bronchial mucus, making it possible to determine the typical time of interaction between the aerosol and the mucus $\left(T_{0}\right)$ and the solubilization period $(\Delta t)$. According to our knowledge it is important to have an experimental technique that permits the monitoring of the rheologic properties of mucus during the interaction with the specific drug.

\section{$\fallingdotseq$ Luis VF. De Oliveira}

Sleep Laboratory, Rehabilitation Sciences Master and Doctoral Degree Program, Nove de Julho University, UNINOVE, São Paulo

Av. Francisco Matarazzo 612, Água Branca, São Paulo-SP CEP: 05001-100, Brazil

email: oliveira.lvf@uninove.br

Data di arrivo del testo: 23/06/2011 - Accettato dopo revisione: 08/07/2011

Multidisciplinary Respiratory Medicine 2011; 6(4): 238-241 


\section{PHOTOACOUSTIC TECHNIQUE (PAT)}

PAT can be applied to study the interaction between different therapeutic aerosols and bronchial mucus. This technique enables the determination of the typical interaction time of solubilization $\left(\mathrm{t}_{0}\right)$ and the solubilization interval $(\Delta t)$ in minutes between aerosol and the sample of secretion. The photoacoustic effect consists in the production of a sound due to the absorption of modulated light that is converted into heat, causing temperature modulation which produces the mechanical effect of periodic expansion and contraction of the sample, originating sound waves that can be detected by a microphone present in the photoacoustic cell (Figure 1). The photoacoustic signal depends on the optical and thermal properties of the sample, which may vary with time. When a sample undergoes changes in its composition or structure, the propagation of heat produced inside is modified, thereby altering the photoacoustic signal [5].

The experimental set-up (Figure 2) used for the thermal effusivity measurements is composed of a 200 W tungsten filament lamp, of which a polychromatic beam is mechanically chopped and focused on the $16 \mu \mathrm{m}$ thick aluminum (Al) foil closing the chamber. The signal from the microphone is connected to a lock-in amplifier (SR, model 850) used to register both the signal and the phase; these are recorded as a function of the modulation frequency. The liquid sample is placed inside a $0.50 \mathrm{~mm}$ thick acrylic ring of $3 \mathrm{~mm}$ inner diameter, whose bottom is closed by a $16 \mathrm{~mm}$ thick $\mathrm{Al}$ foil disk. The entire sample holder is placed on the top of the microphone and is attached by means of a thin layer of vacuum grease [4]. To measure the rheologic properties of human bronchial mucus by PAT, three $5 \mathrm{ml}$ sputum samples per patient are required. These should be spontaneously collected from each selected patient on three consecutive days in a universal collector covered with sterile gauze to absorb any excess saliva. They are then placed in a polystyrene tube lubricated with liquid vaseline to avoid dehydration and stored at $-20^{\circ} \mathrm{C}$ until photoacoustic analysis. Each sample is naturally thawed at room temperature and subsequently submerged in xylol for 5 seconds to remove the liquid vaseline. Then they are divided into three portions with volumes of $0.1 \mathrm{~mL}$ which undergo photoacoustic analysis in a double-blind manner. All the mucus samples must be analyzed by routine laboratory tests based on culture of microorganisms to rule out the presence of pulmonary infection.

In our preliminary study, we performed photoacoustic analyses according to the protocol described by Dumas et al. [3]. Each sputum sample was evaluated for a period of $5 \mathrm{~min}$ to measure the baseline photoacoustic signal.

Subsequently, it was individually nebulized using the aerosols and the solubilization was evaluated by means of monitoring the amplitude of photoacoustic analyses over time. The evolution over time of photoacoustic analyses was adjusted using the Boltzmann equation given by:

$$
P A(t)=\frac{A_{1}-A_{2}}{1+e^{\frac{t-t_{0}}{\Delta t}}}+A_{2}
$$

where $P A(t)$ is the amplitude of the photoacoustic analyses signal at time $\mathrm{t}, \mathrm{A} 1$ and $\mathrm{A} 2$ are the baseline and final amplitudes of the photoacoustic signals, respectively, $t_{0}$ is the time to reach the maximum rate of change in the process and $\Delta t$ is the effective time interval corresponding to the solubilization process. Figure 3 shows the $t_{0}$ and $\Delta t$ parameters and the standard curve for the adjustment of the photoacoustic signal which is produced by a computer program.

\section{PRELIMINARY RESULTS}

In our preliminary study, conducted according to Dumas et al. [3], mucus was obtained from 11 volunteers, divided into two groups: five patients presenting respiratory impairment symptoms (group I) and six healthy subjects (group II). The photoacoustic signal of the mucus absorbing the isotonic saline solution was monitored as a function of time, with measurements being performed each $10 \mathrm{~min}$, up to $120 \mathrm{~min}$. A characteristic time for the half saturation of the absorption process was obtained. The mean values and standard deviations obtained for parameters $t_{0}$ and $\Delta t$ were $23.3 \pm 5.3 \mathrm{~min}$ and $4.5 \pm$ $1.9 \mathrm{~min}$ for group $\mathrm{I}$, and $55.0 \pm 7.7 \mathrm{~min}$ and $16.6 \pm$ 3.4 min for group II respectively, presenting a significant difference (Student's $t$-test, $\mathrm{p}<0.05$ ).

This study has shown that human bronchial mucus from subjects with different health status presents different rheological properties and characteristic times for absorption of the isotonic saline solution. The results confirm the feasibility of using PAT on the study of the solubility behavior of human bronchial mucus after specific therapeutic aerosols.

FIGURE 1: DIAGRAM OF THE PHOTOACOUSTIC CELL USED IN THIS STUDY

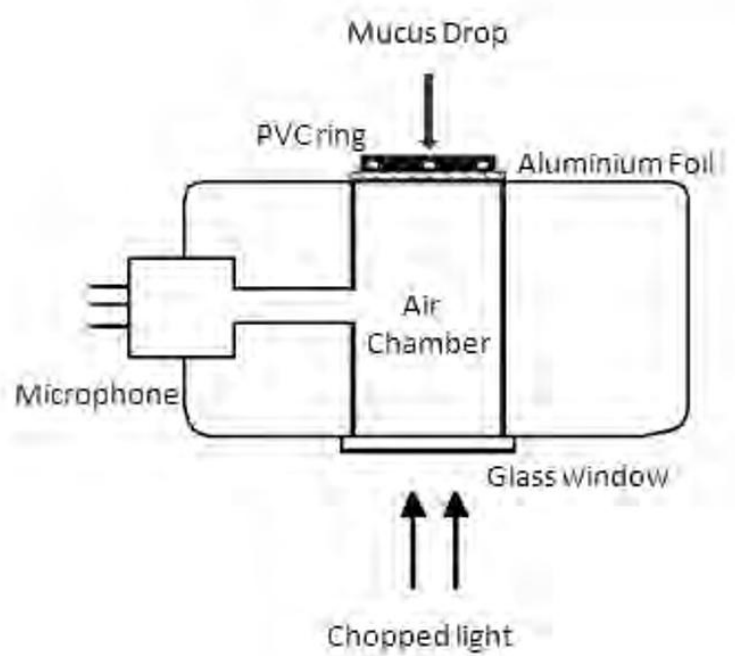




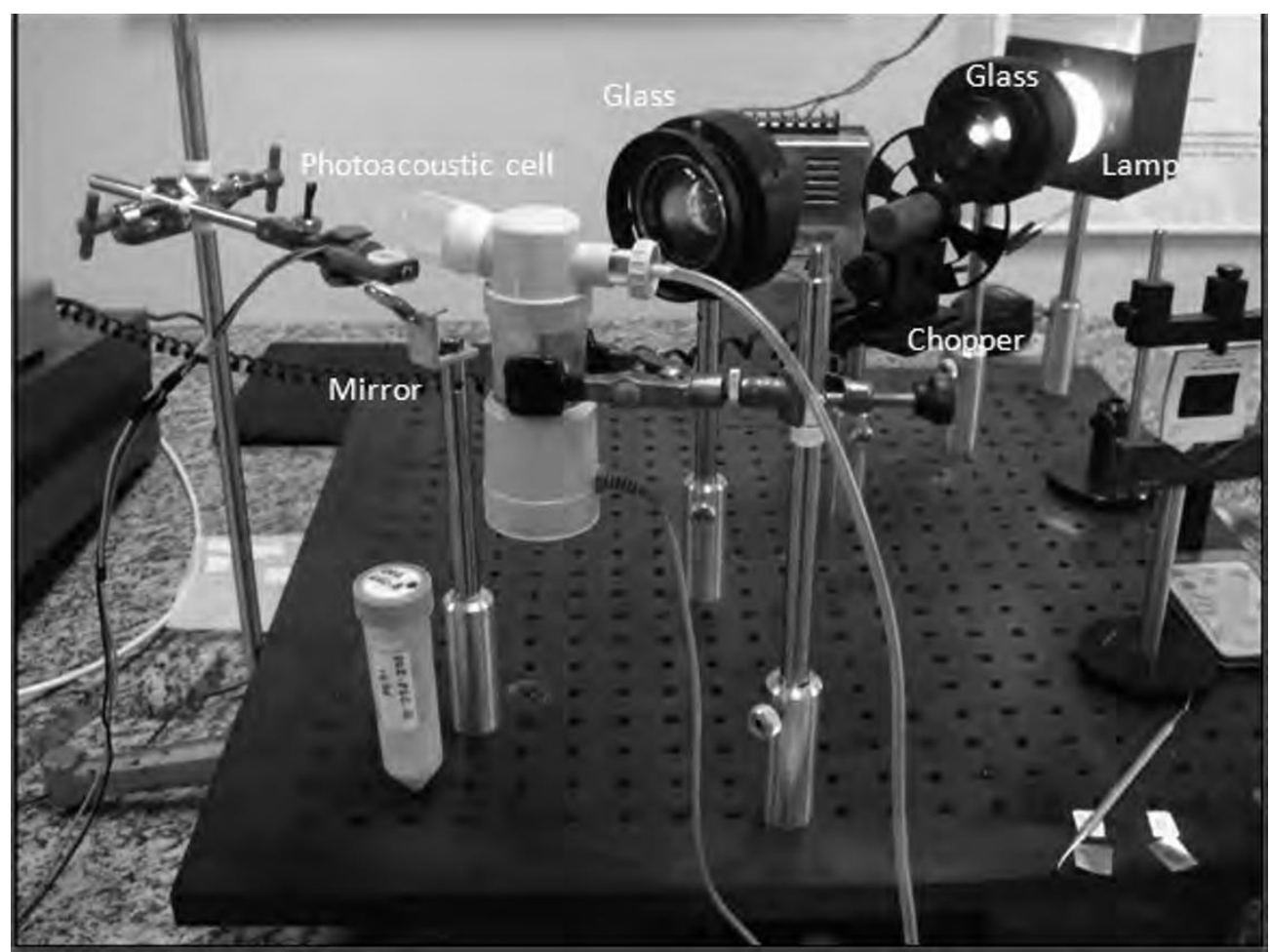

\section{CONCLUSIONS}

A number of diseases of the chest are characterized by increased mucus production, including chronic obstructive pulmonary disease (COPD), bronchiectasis and cystic fibrosis, which, in concert with

FIGURE 3: ADJUSTMENT OF THE PHOTOACOUSTIC SIGNAL (PA) FOR THE TYPICAL INTERACTION TIME $\left(T_{0}\right)$ AND SOLUBILIZATION INTERVAL $(\triangle T)$

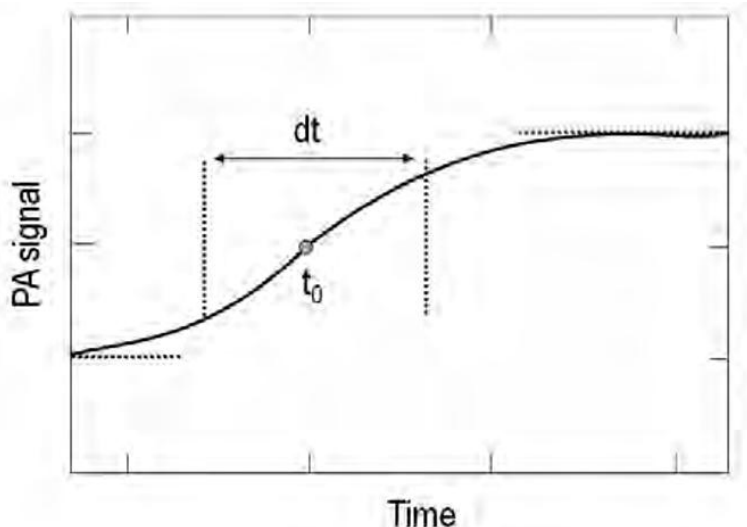

damage to cilia from a variety of host and bacterial sources, can lead to impairment of the mucociliary escalator and retention of secretions within the bronchial tree. Secretion retention can also contribute to airflow obstruction, wheeze, shortness of breath and cough, and the poor clearance of airway secretions promotes recurrent cycles of pulmonary infection and inflammation. Mucociliary clearance, the process in which mucus together with substances trapped within are moved out of the lungs, is an important defense mechanism of human body. Drugs may alter this process, such that it is necessary to know the effect of these drugs on mucociliary clearance. PAT measurements as a function of time permit to characterize the solubilization kinetics of pulmonary secretions, and to know the effects of mucolytic drugs in samples of mucus derived from different respiratory pathologies.

ACKNOWLEDGMENTS: The authors thank Fundação de Amparo à Pesquisa (Fapesp, acronym in Portuguese) and Conselho Nacional de Desenvolvimento Científico e Tecnológico (CNPq, acronym in Portuguese) for supporting this research.

CONFLICT OF INTEREST STATEMENT: The authors declare that there is no associaton with any commercial enterprise that has interest in the object of this study. 


\section{References}

1. Macchione M, Guimarães ET, Saldiva PH, Lorenzi-Filho G. Methods for studying respiratory mucus and mucus clearance. Braz J Med Biol Res 1995;28:1347-1355.

2. King M. Experimental models for studying mucociliary clearance. Eur Respir J 1998;11:222-228.

3. Dumas FL, Marciano FR, Oliveira LV, Barja PR, AcostaAvalos D. Photoacoustic monitoring of the absorption of isotonic saline solution by human mucus. Med Eng Phys 2007;29:980-983.

4. Balderas-López JA, Cruz Orea A, Acosta-Avalos D, Alvarado
Gil JJ, Zelaya O, Sànchez F, Falcony C, Vargas H. Photoacoustic measurements of transparent liquid samples: thermal effusivity. Measurements Science and Technology 1995;6:1163-1168.

5. Barboza MAI, Mattos CCB, Barja PR, Oliveira LVF, Mattos LC. Influence of secretor and non secretor phenotypes on the solubilization of the pulmonary mucus by three common medicines in cystic fibrosis patients assessed using photoacoustic analysis. Arch Med Sci 2008;4:386-391. 